\title{
Perception, par les infirmières en oncologie, de leurs relations avec les proches des patients dans un milieu de soins ambulatoires en oncologie: une étude multi-méthodes
}

par Michelle Lobchuk et Sonia Udod

\begin{abstract}
Abrégé
L'examen des tendances révèle la hausse du nombre de personnes recevant un diagnostic de cancer et y survivant ainsi qu'un recours accru aux services de santé ambulatoires en oncologie et aux soins dispensés par les aidants naturels. Malgré cette tendance, peu de recherches ont porté sur les expériences des infirmières en matière de soutien à fournir aux proches qui prennent soin de patients dans la communauté. La satisfaction au travail des infirmières en oncologie a connu une baisse importante dans les milieux de soins ambulatoires puisqu'elles s'inquiètent de ne pas pouvoir fournir-de façon constante-des soins sécuritaires et optimaux aux patients et à leurs proches. Quoique d'autres études rapportaient que le manque de temps et de ressources sont des aspects malheureusement acceptés des environnements de travail des infirmières, notre étude multiméthodes à petite échelle s'intéressait à la manière dont les environnements de travail peuvent encore répondre au besoin croissant de rehausser, dans les soins ambulatoires en oncologie, le soutien et les relations entre infirmières, patients et proches.
\end{abstract}

À la lumière du virage ambulatoire en cancérologie, on demande aux infirmières en oncologie de fournir un enseignement efficace aux patients et à leurs familles relativement aux principes guidant le soutien et les soins aux personnes atteintes de cancer (Association canadienne des infirmières en oncologie [ACIO], 2001). Les infirmières doivent non seulement évaluer les problèmes de santé actuels et éventuels des personnes qu'elles soignent mais encore s'occuper du système patient-famille puisque ce dernier influence les besoins de santé des patients de nature sociale, économique, physique, spirituelle et émotionnelle (ACIO, 2001). Bakker, Fitch, Green, Butler et Olson (2006) ont révélé qu'en dépit des défis de taille auxquels elles font face dans leur vie professionnelle actuelle, les infirmières en oncologie sont capables de poursuivre leur travail, «pour l'instant» (p. 84, traduction libre). Les résultats les plus récents suggèrent fortement que les environnements de travail doivent soutenir les infirmières en oncologie tandis qu'elles s'efforcent de trouver un juste équilibre entre, d'une part, les aspects cliniques ou plus techniques de leur rôle dans les milieux de soins ambulatoires et, d'autre part, la capacité à préserver les vitales relations infirmière-patient-famille (Mcilfatrick, Sullivan \& McKenna, 2006; McLeod, Tapp, Moules \& Campbell, 2010).

Les relations que les infirmières établissent dans le lieu de travail avec des collègues infirmières et d'autres intervenants de la santé

\section{Au sujet des auteures}

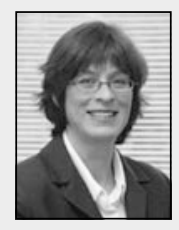

Michelle Lobchuk, inf., Ph.D., professeure agrégée, Université du Manitoba, Faculté des sciences infirmières, Room 315, 89 Curry Place, Winnipeg, $M B$ R3T 2N2

Tél. : (204) 474-7135, Téléc. : (204) 474-7682; Courriel : Michelle_Lobchuk@umanitoba.ca

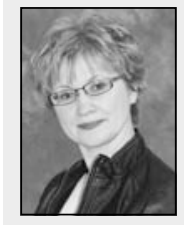

Sonia Udod, inf., M.Sc.inf., Ph.D.(c), Université de la Saskatchewan, Collège des sciences infirmières, Saskatoon, SK jouent un rôle essentiel dans leur satisfaction au travail (Baker et al., 2010, p. 62). Toutefois, les relations entre l'infirmière et les soignants membres de la famille constituent un élément fréquemment négligé de la satisfaction des infirmières à l'égard de leur qualité de vie professionnelle (Laschinger \& Finegan, 2005; Laschinger, Almost \& Tuer-Hodes, 2003). Eriksson (2001) et Lindholm, Makela, RantanenSiljamaki et Nieminen (2007) ont rapporté que les soignants membres de la famille évaluent le contenu des soins et la manière dont ceux-ci sont prodigués notamment les compétences professionnelles, la fiabilité des divers membres du personnel et l'innocuité des soins. La frustration ressentie par les soignants membres de la famille provient souvent du faible nombre d'intervenants de la santé et du manque d'encouragement à s'impliquer dans la prise de décision partagée concernant les soins aux patients (Flanagan, 2001; Isikhan, Comez \& Danis, 2004; Tamayo, Broxson, Munsell \& Cohen, 2010; Vivar, Canga, Canga \& Arantzamendi, 2009). Dans leur récente étude qualitative, McLeod, Tapp, Moules et Campbell (2010) décrivaient la réaction d'un proche qui se sentait effrayé et isolé dans un contexte de soins qu'il percevait comme étant impersonnel, indifférent et insensible. Ce proche commentait que les infirmières devaient fournir les aspects relationnels des soins dans un environnement de travail infirmier aux nombreuses contraintes de temps et orienté sur les tâches, un défi qui débouchait, chez lui, sur des sentiments de frustration et de crainte. Il est généralement reconnu que les soignants membres de la famille peuvent se sentir dépassés par les pressions associées à la prestation des soins à leurs proches, un sentiment qui peut être aggravé par leurs perceptions d'un manque de soutien relationnel de la part des infirmières. C'est ce qu'affirment Greco, Laschinger et Wong (2006) lesquels ont indiqué que les structures organisationnelles au sein du lieu de travail façonnent les expériences de travail des infirmières et que la suppression des barrières existant entre les infirmières et les personnes qu'elles soignent facilite la pratique professionnelle des soins infirmiers. Ceci souligne l'importance de bien saisir les facteurs qui, dans l'environnement de travail des infirmières, peuvent avoir une incidence sur le vécu des infirmières et sur les résultats pour les patients (McGillis Hall, Doran \& Pink, 2008).

Plusieurs raisons convaincantes viennent justifier la présente enquête. Le cancer est une maladie chronique qui peut avoir des impacts à long terme sur les patients et les familles. Selon les statistiques 2010 de la Société canadienne du cancer, il y a eu, entre 2009 et 2010, une augmentation de 1,6 \% des nouveaux cas de cancer et une augmentation de 1,2 \% des décès par cancer qui sont attribuées à la croissance et au vieillissement de la population du Canada (Société canadienne du cancer, Comité directeur, 2010). L'Agence de la santé publique du Canada (2004) estimait que d'ici 2020, le nombre de patients atteints de cancer sous traitement au Canada aura doublé par rapport à 2001. Il est évident que les prévisions concernant l'incidence croissante du cancer et la réalité de la croissance et du vieillissement de la population atteinte de cancer ont des implications majeures pour les infirmières œuvrant en milieu ambulatoire. Bien que les politiques des établissements de soins du Canada visent à améliorer les conditions de travail des infirmières, celles qui travaillent en première ligne, notamment les infirmières en oncologie, constatent que la qualité de leur vie professionnelle ne s'améliore guère (Priest, 2006; Torgerson, 2007). Le Canada fait face à une grave pénurie de personnel infirmier qui menace de mettre en péril les soins fournis aux habitants, y compris ceux qui sont atteints de cancer et leurs proches (O’Brien-Pallas et al., 2005). La prévalence et 
la chronicité du cancer, le manque d'amélioration de la qualité de la vie au travail des infirmières et la pénurie de personnel infirmier constituent des obstacles à la réalisation de l'objectif voulant que les familles soient de véritables "partenaires de soins». Les perceptions des infirmières sur le rôle des familles en tant que clients, ressources et/ou collaboratrices dans la planification et la prestation des soins aux patients n'ont pas été explorées dans les milieux de soins ambulatoires en cancérologie. Étant donné que les soignants membres de la famille sont des partenaires essentiels des soins aux patientspuisqu'ils ont souvent une connaissance experte du vécu de la maladie par le patient, de sa situation sociale, de ses comportements, attitudes, préférences et même de ses facteurs de risque (Hobbs \& Sodomka, 2000), les administrateurs des soins de santé doivent trouver des manières valables de soutenir les infirmières en facilitant la prestation de soins aux patients à la fois sécuritaires et efficaces ainsi que la prestation de soutien aux soignants membres de la famille. Les milieux de travail positifs profitent aux infirmières en oncologie et, en bout de ligne, à la qualité des soins qu'elles dispensent aux personnes diagnostiquées d'un cancer et à leurs proches.

Dans cette étude exploratoire descriptive, nous nous sommes demandées: (1) Quelles sont les perceptions des infirmières en oncologie à l'égard de leurs soins et de leurs relations avec les patients ayant le cancer et leurs proches dans un milieu de soins ambulatoires en oncologie?; (2) En quoi les perceptions des infirmières en oncologie à l'égard de leurs soins et de leurs relations avec les patients et leurs proches ont-elles un effet incitatif ou dissuasif sur la satisfaction des infirmières relativement à la qualité de leur vie professionnelle?; (3) Quelles sont les perceptions des infirmières en oncologie à l'égard de leur environnement de travail au niveau des soins et des relations qui les lient, aux patients et aux proches?

\section{Méthodologie}

Il s'agissait d'une étude exploratoire descriptive faisant appel à des méthodes d'étude quantitatives et qualitatives et à un échantillon choisi à dessein comprenant neuf infirmières qui œuvrent dans un centre de cancérologie d'une province de l'ouest canadien, dans des cliniques axées sur divers sièges de cancer. L'approbation éthique a été obtenue du comité d'éthique de la recherche en éducation/sciences infirmières et l'approbation d'accès au personnel a été accordée par le comité des incidences sur les ressources du centre de cancérologie. L'infirmière chef nous a invitées à rencontrer les infirmières des cliniques durant leurs réunions d'études de cas à deux sites et de les inviter à participer à l'étude. Des copies d'une lettre d'invitation ont été remises aux infirmières; celles-ci étaient priées d'indiquer si elles acceptaient ou non de participer à l'étude. L'infirmière chercheuse a ramassé les lettres d'invitation puis a contacté les infirmières qui avaient accepté de participer afin d'organiser une entrevue de groupe après le travail à une heure et une date convenant aux participantes dans chacun des deux sites de recrutement. Dans ces deux sites, les infirmières ont accepté d'assister à leur groupe de discussion respectif immédiatement après leur journée de travail et ce, dans une salle de conférence située en dehors de leur établissement dans un cas et dans la salle de réunion du personnel, dans l'autre. En gage d'appréciation du temps et des efforts consacrés à notre étude, nous avons fourni aux participantes un honoraire de 25 \& et un repas complet avant le commencement des entrevues de groupe. L'infirmière chercheuse posait les questions de l'entrevue de groupe et la première auteure (ML) assistait aux entrevues de groupe à titre de participante-observatrice. Afin de veiller au bon fonctionnement de l'appareil d'enregistrement sonore, une assistante de recherche a également assisté à la première entrevue de groupe mais n'a aucunement participé au processus de collecte des données.

Après avoir obtenu le consentement éclairé des participantes, l'infirmière chercheuse leur a demandé de remplir a) un questionnaire démographique sur le personnel infirmier élaboré par l'équipe de recherche et comprenant 18 questions, b) un questionnaire
Activités infirmières axées sur la communication avec les proches (Activités), comprenant 17 questions et une échelle en 4 points (3, Oui, j'ai fait ça; 2, Oui, une autre infirmière a fait ça; 1 , Non, pas fait ou ne sais pas; 0, Ne s'applique pas aux proches ici), c) un questionnaire Obstacles aux soins pour les infirmières (Obstacles), comprenant 14 questions (questions exigeant une réponse «Oui» ou «Non») et enfin, d) un questionnaire Répondre aux besoins des prochesQuestionnaire de l'infirmière (Satisfaction) comprenant 3 questions et une échelle à 11 points (allant de 0 , Pas du tout satisfaite à 10 , Très satisfaite). Ces outils avaient été élaborés à l'origine pour saisir les perceptions des infirmières au niveau de l'aide apportée aux proches des patients d'unités de soins intensifs (Downey, Engelberg, Shannon $\&$ Curtis, 2006). Afin d'assurer la pertinence et la clarté des questions démographiques sur le personnel infirmier, des questions des instruments et des questions de l'entrevue de groupe, nous avons consulté l'infirmière chef, une infirmière en pratique avancée et une infirmière d'une des cliniques du centre de cancérologie. Plusieurs révisions mineures ont été apportées afin d'intégrer des possibilités de réponse appropriées et ce, uniquement dans l'outil démographique.

Une fois le remplissage des questionnaires terminé, l'infirmière chercheuse a dirigé le groupe de discussion-soumis à un enregistrement sonore-selon le guide d'entrevue conçu par l'équipe de recherche. Les questionnaires et les entrevues de groupe réalisées auprès de deux groupes (groupe $1, n=7$; groupe $2, n=2$ ) ont pris deux heures. Après que l'infirmière chercheuse a parlé aux infirmières ayant exprimé leur désir de participer à l'étude, nous projetions avoir huit infirmières dans le premier site et trois dans le second qui participeraient aux entrevues de groupe. Nous n'avons pas pu déterminer les raisons pour lesquelles deux infirmières ne se sont pas présentées. Quoique l'échantillon soit de faible taille pour le second groupe de discussion, nous avons décidé de le tenir malgré tout, étant donné que ces infirmières pouvaient s'exprimer sur leurs relations avec les proches dans le cadre de leur rôle infirmier et dans l'environnement de travail existant dans leur site.

La statistique descriptive a été employée afin de saisir les caractéristiques sociodémographiques des participantes et leurs réponses aux questionnaires. L'analyse de contenu a été utilisée pour aborder les questions 1 à 3 de l'étude. L'enregistrement audio des contributions des infirmières aux questions du guide d'entrevue semi-dirigée a été transcrit, et la transcription a été analysée par deux chercheuses (ML et SU) ainsi qu'une assistante de recherche au moyen de l'analyse de contenu et de techniques de comparaison constante en vue de dégager, coder, catégoriser, classer et nommer les caractéristiques au sein des données (Lincoln \& Guba, 1985; Patton, 2002; Strauss \& Corbin, 1998).

\section{Résultats}

Le tableau 1 permet de constater que tous les participants étaient en fait des infirmières de race blanche. La majorité d'entre elles avaient entre 35 et 44 ans et avaient obtenu un baccalauréat ou avaient fait des études de cycle supérieur. La plupart des infirmières participant à l'étude avaient obtenu leur certification en soins infirmiers en oncologie. Soixante-dix-huit pour cent d'entre elles occupaient un emploi à plein temps permanent et toutes faisaient le quart de jour de huit heures. Les participantes travaillaient en éducation clinique, au bureau des recherches cliniques ou dans divers domaines cliniques dont les suivants : maladies hématologiques bénignes, thorax, greffe de moelle osseuse, oncologie chirurgicale, sphère tête et cou ou sites cliniques multiples. Le jour de la tenue de leur groupe de discussion respectif, huit infirmières ont signalé avoir travaillé huit heures. Au cours des sept jours précédents, sept infirmières avaient travaillé de 35 à 40 heures et deux autres, entre 40 et 45 . Dans leur majorité, elles travaillaient depuis un an ou plus dans leurs actuelles cliniques de cancérologie respectives et œuvraient principalement auprès d'oncologues médicaux dans la dispensation des soins aux patients; une infirmière a indiqué qu'elle travaillait avec des oncolo- 
gues médicaux et des hématologues; de plus, deux infirmières ont mentionné qu'elles travaillaient avec des oncologues médicaux, des radio-oncologues et des chirurgiens-oncologues. La majorité des participantes occupaient un poste d'infirmière depuis 10 à 35 ans.

Dans l'échelle Activités (tableau 1), 100 pour cent des infirmières participant à l'étude confirmaient qu'elles effectuaient les 8 activités avec les proches suivantes, sur 17 : elles expliquaient l'équipement médical, discutaient des choses de la vie que le patient valorisait, de la maladie et de son traitement, des émotions du patient, évoquaient des souvenirs liés au patient, rassuraient les proches qu'ils pouvaient parler à leur être cher et le toucher, discutaient de ce que le patient désirait et assuraient aux proches que le patient allait être confortable. Le sujet ayant reçu le moins de confirmations était la discussion des besoins spirituels ou religieux des familles quoique la majorité ait indiqué prendre en charge ce besoin des proches. Dans l'échelle Obstacles (tableau 2), la majorité des infirmières sanctionnaient les questions suivantes comme étant des obstacles à la satisfaction des besoins des familles : s'occuper de proches éprouvant de la colère, les attentes non réalistes, le manque d'intimité, les problèmes de langue et le nombre insuffisant d'infirmières. Aucune participante n'a mis la politique en matière de visites au rang des obstacles, et une seule participante a retenu comme obstacle le manque de soutien de la part du personnel infirmier ou de travail social. Les enjeux les moins appuyés à titre d'obstacles par les participantes étaient les suivants : les médecins qui décourageaient la communication avec les proches, les proches qui ne faisaient pas de visite ni ne plaçaient d'appels, les patients qui sont trop gravement malades, les conflits avec les médecins, et enfin, s'occuper des proches est à l'extérieur du champ de pratique. À l'intérieur d'une étendue théorique allant de 0 à 10 unités, les résultats moyens aux trois questions figurant dans l'échelle de satisfaction étaient: satisfaite que les infirmières répondaient aux besoins émotionnels des proches $(\mathrm{m}=6,77$, écart-type $=1,98)$, à leurs besoins physiques $(m=7,33$, écart-type $=2,06)$ et que l'équipe de soins de santé répondait aux besoins des proches $(\mathrm{m}=7,0$, écarttype $=1,66$ ). Le résultat moyen à l'échelle de satisfaction (totale) s'élevait à 7,04 (écart-type=1,46; étendue théorique : satisfaite de 0 à 10).

\section{Résultats des groupes de discussion}

Plusieurs thèmes fondamentaux sont ressortis de cette étude pilote. Des facteurs positifs et des facteurs négatifs influencent les relations des infirmières avec les patients et leurs proches et ce, dans leurs environnements de travail.

Facteurs liés au lieu de travail. Les participantes rapportaient que leur environnement structurel ne leur fournissait pas les conditions nécessaires à la prestation de soins efficaces aux patients et à leurs proches. Les participantes faisaient état du manque de temps, du manque d'espace et parfois même du manque de contrôle sur leur environnement de travail, ce qui les empêchait de répondre adéquatement aux besoins de santé des patients et de leurs proches. Les infirmières mentionnaient presque toutes un manque d'espace pour rencontrer les patients et leurs proches et leur fournir l'intimité et le confort dont ils avaient besoin pour les aider à s'adapter efficacement à des situations potentiellement mortelles. Une participante l'a exprimé de la façon suivante :

Ouais, et je me demande dans quelle mesure cela peut bien les aider à être suffisamment à l'aise pour poser des questions quand la salle est pleine d'inconnus, je pense que, parfois, ils attendent un peu trop longtemps avant de m'en poser une sur une chose particulière, peut-être parce qu'ils ne veulent pas la poser devant tout le monde.

Les participantes ont également décrit leur lieu de travail comme un endroit qui manquait fréquemment des ressources physiques et humaines permettant de répondre de manière satisfaisante aux besoins de santé des patients et de leurs proches. Les ressources

\begin{tabular}{|c|c|c|}
\hline Caractéristique & $\mathrm{N}^{\text {bre }}$ d'infirmières & $\%$ \\
\hline Sexe : féminin & 9 & 100 \\
\hline Âge : $35-44$ ans & 6 & 67 \\
\hline $45-64$ ans & 3 & 33 \\
\hline Race blanche & 9 & 100 \\
\hline \multicolumn{3}{|l|}{ Formation } \\
\hline Diplôme d'hôpital & 2 & 22 \\
\hline Diplôme collégial en soins infirmiers & 1 & 11 \\
\hline Baccalauréat en sciences infirmières & 2 & 22 \\
\hline Baccalauréat dans un autre domaine & 2 & 22 \\
\hline Maîtrise en éducation & 1 & 11 \\
\hline Donnée manquante & 1 & 11 \\
\hline \multicolumn{3}{|l|}{ Nombre d'années en soins infirmiers } \\
\hline$\geq 6$ et $<10$ ans & 2 & 22 \\
\hline$\geq 10$ et $<15$ ans & 3 & 33 \\
\hline$\geq 15$ et $\leq 35$ ans & 4 & 44 \\
\hline \multicolumn{3}{|l|}{ Certification } \\
\hline $\mathrm{CSIO}(\mathrm{C})$ & 5 & 56 \\
\hline $\operatorname{CSI}(\mathrm{C})$ & 1 & 11 \\
\hline Donnée manquante & 1 & 11 \\
\hline Sans objet & 2 & 22 \\
\hline \multicolumn{3}{|l|}{ Situation d'emploi } \\
\hline Permanent à plein temps & 7 & 78 \\
\hline Permanent à temps partiel & 2 & 22 \\
\hline \multicolumn{3}{|l|}{ Quart de travail normal } \\
\hline Quart de 8 heures, de jour & 9 & 100 \\
\hline \multicolumn{3}{|c|}{ Nombre d'années passées dans la clinique consacrée à un siège donné } \\
\hline$\leq 1$ an & 2 & 22 \\
\hline$>1$ an, $\leq 5$ ans & 4 & 44 \\
\hline$>5$ ans & 2 & 22 \\
\hline Donnée manquante & 1 & 11 \\
\hline \multicolumn{3}{|c|}{ Dans votre clinique, qui dispense les soins médicaux aux patients? } \\
\hline Oncologue médical & 6 & 67 \\
\hline Radio-oncologue & 3 & 33 \\
\hline Chirurgien-oncologue & 1 & 11 \\
\hline Hématologue & 4 & 44 \\
\hline \multicolumn{3}{|c|}{$\mathrm{N}^{\text {bre }}$ d'heures de travail durant les derniers 7 jours } \\
\hline$<35$ heures & 2 & 22 \\
\hline$\geq 35$ heures, $\leq 40$ heures & 5 & 56 \\
\hline$>40$ heures, $<45$ heures & 2 & 22 \\
\hline \multicolumn{3}{|c|}{$\mathrm{N}^{\mathrm{bre}}$ d'heures de travail durant les dernières 24 heures } \\
\hline$<7,75$ heures & 1 & 11 \\
\hline$\geq 7,75$ heures, $\leq 8$ heures & 6 & 67 \\
\hline$>8$ heures & 1 & 11 \\
\hline Donnée manquante & 1 & 11 \\
\hline \multicolumn{3}{|l|}{ Quart de travail effectué aujourd'hui } \\
\hline Quart de jour de 8 heures & 8 & 89 \\
\hline Donnée manquante & 1 & 11 \\
\hline \multicolumn{3}{|l|}{ Poste au centre de cancérologie } \\
\hline Infirmière de chevet & 6 & 67 \\
\hline Éducatrice clinique & 1 & 11 \\
\hline ICS & 1 & 11 \\
\hline Autre rép. & 1 & 11 \\
\hline
\end{tabular}


financières réduites empêchaient souvent les infirmières d'assister à des ateliers pédagogiques en vue de rehausser leurs connaissances et compétences, par exemple :

... comment pouvons-nous continuer à soutenir [les patients], tu sais, quelquefois cela fait du bien d'aller à des séances pédagogiques, mais le plus souvent, on n'a pas le temps ... et il n'y a pas d'argent et il n'y a personne pour te remplacer et c'est aussi un obstacle parce que parfois, que tu y ailles ou non, tu en ressors et tu as l'impression de savoir que tu fais exactement ce qu'ils viennent de te dire de faire, mais maintenant tu sais que tu fais une grosse différence parce qu'ils confirment que tu es en train de faire ce qu'il faut. C'est aussi du soutien, ça.

Les participantes ont également indiqué que le manque de personnel infirmier aggravait leur lourde charge de travail, par exemple :

\begin{tabular}{|c|c|c|c|}
\hline Question & $\begin{array}{l}\text { \% (n) Rép. } \\
\text { cochée }\end{array}$ & $\begin{array}{l}\text { Moyenne } \\
\text { (écart-type) }\end{array}$ & Médiane \\
\hline \multicolumn{4}{|l|}{ ACTIVITÉS } \\
\hline $\begin{array}{l}\text { 1. Expliquer l'équipement } \\
\text { médical }\end{array}$ & $100(9)$ & & \\
\hline $\begin{array}{l}\text { 2. Ce à quoi s'attendre } \\
\text { durant les conférences }\end{array}$ & $89(8)$ & & \\
\hline $\begin{array}{l}\text { 3. Besoins spirituels ou } \\
\text { religieux des proches }\end{array}$ & $67(6)$ & & \\
\hline $\begin{array}{l}\text { 4. Intervention pour aborder } \\
\text { les besoins spirituels/ } \\
\text { religieux }\end{array}$ & $78(7)$ & & \\
\hline $\begin{array}{l}\text { 5. Besoins culturels } \\
\text { particuliers }\end{array}$ & $89(8)$ & & \\
\hline $\begin{array}{l}\text { 6. Intervention pour aborder } \\
\text { les besoins culturels }\end{array}$ & $78(7)$ & & \\
\hline $\begin{array}{l}\text { 7. Ce que le patient valorise } \\
\text { dans la vie }\end{array}$ & $100(9)$ & & \\
\hline $\begin{array}{l}\text { 8. Maladie et traitement du } \\
\text { patient }\end{array}$ & $100(9)$ & & \\
\hline $\begin{array}{l}\text { 9. Discuter des émotions } \\
\text { avec la famille }\end{array}$ & $100(9)$ & & \\
\hline $\begin{array}{l}\text { 10. Évoquer des souvenirs } \\
\text { liés au patient }\end{array}$ & $100(9)$ & & \\
\hline $\begin{array}{l}\text { 11. OK de parler à leur être } \\
\text { cher et de le toucher }\end{array}$ & $100(9)$ & & \\
\hline $\begin{array}{l}\text { 12. Discuter de ce que les } \\
\text { patients peuvent désirer }\end{array}$ & $100(9)$ & & \\
\hline $\begin{array}{l}\text { 13. Trouver un endroit privé } \\
\text { pour les proches }\end{array}$ & $78(7)$ & & \\
\hline $\begin{array}{l}\text { 14. Désaccord entre les } \\
\text { proches }\end{array}$ & $78(7)$ & & \\
\hline $\begin{array}{l}\text { 15. Changements dans le } \\
\text { plan de soins du patient }\end{array}$ & $78(7)$ & & \\
\hline $\begin{array}{l}\text { 16. Soutien des décisions } \\
\text { prises par les proches } \\
\text { concernant les soins }\end{array}$ & $78(7)$ & & \\
\hline $\begin{array}{l}\text { 17. Assurer aux proches que } \\
\text { le patient sera confortable }\end{array}$ & $100(9)$ & & \\
\hline Total pour l'échelle Activités & & $15,33(1,94)$ & 16,00 \\
\hline
\end{tabular}

Ce qui se produit chez les infirmières chercheuses de notre département est que, très souvent, nous finissons par aller bien au-delà de ce qu'il y a dans notre description d'emploi ... Nous sommes censées être les coordonnatrices de l'étude, réaliser l'essai, obtenir le consentement des patients, effectuer les examens pour qu'ils puissent participer à l'essai et faire le suivi de leur traitement tout au long de l'essai et nous concentrer quand nous sommes avec les patients, classer leurs toxicités et obtenir tous leurs médicaments et faire toute la documentation afférente. Mais le problème est que nous sommes toutes passées par le domaine clinique auparavant, nous avons ça dans le sang et nous avons toutes tendance à prendre en charge le rôle de l'infirmière en soins intégraux, quelque chose que nous ne sommes pas censées faire. Je tape toujours sur la main des autres infirmières quand elles font ca, puis je me surprends à faire comme elles, parce que la chose est que nous n'en avons tout simplement pas le temps ... Nous avons soixante-deux essais ouverts en ce moment et nous n'avons pas assez de personnel pour bien nous en occuper.

Pourtant, les participantes mentionnaient leur dépendance positive vis-à-vis d'autres infirmières et de membres de l'équipe de soins de santé et s'étaient méritées la confiance des médecins sur le plan de la satisfaction des besoins des patients et de leurs proches. Les infirmières s'appuyaient sur ces relations de soutien pour faciliter la coordination et la satisfaction des besoins de santé des patients et de leurs proches dans la clinique de cancérologie. En outre, les participantes parlaient en bien d'un groupe de soutien conçu pour aider les infirmières à composer avec les événements potentiellement traumatisants tels que le décès d'un patient. Fait plus important encore, les relations de soutien avec les collègues jouaient un rôle capital dans le rehaussement de la qualité de la vie professionnelle des infirmières et leur permettaient de s'attaquer plus efficacement aux défis qu'elles rencontraient chez les patients en oncologie et leurs proches. Malgré les carences de leur environnement physique, les infirmières établissaient et maintenaient des relations avec des collègues et celles-ci leur fournissaient la force nécessaire pour diriger leur attention sur l'unité patient-famille.

Facteurs liés aux patients/aux proches. Les participantes disaient des proches qu'ils manifestaient une gamme d'émotions et de comportements et elles dressaient le portrait suivant des familles : ne s'impliquent aucunement dans le traitement du patient, ne souhaitent pas recevoir d'information, ne posent pas de questions, et enfin, refusent l'aide des divers membres de l'équipe de soins de santé. L'incapacité de certaines participantes à aider les patients/ proches ne venait pas nécessairement d'un manque de compétence mais plutôt du fait que les patients/proches ne voulaient pas accéder aux services de soutien offerts par d'autres membres de l'équipe de soins de santé. Lorsque cela se produisait, les infirmières assumaient la tâche supplémentaire d'aborder et de soulager l'angoisse que les patients et leurs proches ressentaient, par exemple :

Je pense que, des fois, il faut que tu fasses tout pour tes patients; il arrive que les patients ne veulent pas qu'on les aiguille vers qui que ce soit, surtout s'ils ont des enjeux d'ordre mental et qu'ils ressentent une angoisse continue. Parfois, ces patients ne veulent pas du tout qu'on les réfère à un autre intervenant à des fins d'assistance et il arrive que tu te sentes coincée à cause des contraintes temporelles, parce que tu sais que leurs besoins sont bien trop épuisants pour toi [...] que la somme totale des ressources que tu dois consacrer à cette personne te dépasse et que tu ne peux pas les référer.

En dépit de la valeur et du soutien importants accordés à l'engagement actif des proches dans les soins du patient, les participantes rapportaient que ce processus était, pour elles, à la fois lourd 
et dévoreur de temps. Des problèmes survenaient lorsque de nombreux proches d'un même patient contactaient l'infirmière à propos de son plan de traitement ou lorsque les distances entre les proches les empêchaient d'interagir et de véhiculer des renseignements clés sur le plan de traitement du patient; par exemple :

Mais la distance peut aussi entrer en jeu parce que les proches qui vivent plus loin peuvent se sentir impuissants, qu'ils ne sont pas dans le coup et n'apportent aucune contribution, cela signifie passer du temps avec des proches [qui] ne sont peutêtre pas dans la clinique mais qui font partie de la famille.

Selon les participantes, que les proches participent activement ou non aux soins au patient, les attentes du patient et de ses proches étaient fréquemment incompatibles avec le temps, l'énergie, les connaissances ou les compétences dont disposaient les infirmières. En dépit de ces contraintes, les participantes consacraient une quantité considérable de leur temps et de leur énergie à réconforter les patients et à faire preuve de sensibilité dans leur satisfaction des besoins des patients/familles en regard de leur phase d'adaptation au diagnostic de cancer. Essentiellement, les infirmières se caractérisaient en disant qu'elles jonglaient avec les multiples besoins et exigences des proches et des patients et qu'elles consacraient une partie importante de leur temps à s'occuper des questions relationnelles.

Satisfaction à l'égard de leur qualité de vie professionnelle. Les participantes affirmaient dans un même élan unanime que les stimulantes activités leur permettant d'établir et d'entretenir des relations de confiance avec les patients et leurs proches jouaient un rôle capital sur le plan de la qualité de leur vie au travail. Établir des rapports, composer avec des circonstances difficiles sur le plan de la dynamique patient-famille, s'impliquer dans des contacts réguliers et constater les progrès post-traitement et post-deuil étaient décrits comme faisant partie intégrante de leur qualité de vie au travail. Trois participantes ont partagé leurs perspectives :

Parce qu'on est assis ensemble, des fois [durant] le premier cycle, on est assis ensemble et on bavarde un peu pour soulager la tension, et tu apprends des choses au sujet de leur famille, du nombre d'enfants qu'ils ont, d'où ils sont originaires et de causer un brin comme ça, ça les aide à se relaxer... puis ils te posent des questions sur ta propre famille, ce qui fait qu'on finit par se connaître les uns les autres. Je ne dis pas que c'est tout le monde, mais un grand nombre quand même.

C'est drôle [...] parce que nous sommes un petit groupe [...] je crois qu'ils ont l'impression de bien nous connaitre parce qu'ils ont une si grande confiance en nous.

Je les aime parce que, pour moi, c'est tout un défi de les faire descendre de ce niveau-ci à ce niveau-là, faites les donc venir tous ceux qui sont dérangés ou [ceux] qui sont vraiment stressés et remplis de colère et ils veulent venir ici ...et ils vont te dire de quoi il retourne et voilà ce qui va se produire... j'adore ça...

Aider les patients et leurs proches à s'y retrouver dans les options de traitement et dans les changements systémiques exige temps et dévouement et pourtant, il était stimulant et gratifiant pour les participantes d'aider l'unité patient-famille à mieux s'adapter aux soins oncologiques. Les participantes éprouvaient de la satisfaction envers leur travail quand des marques de reconnaissance verbales ou écrites de leurs soins infirmiers leur étaient présentées par leurs collègues, leurs patients et/ou les proches. Les participantes déclaraient se sentir respectées et valorisées pour les connaissances et les compétences qu'elles mettaient en œuvre auprès de cette population spécialisée, ce qui influençait positivement leurs relations avec les patients et leurs proches.

Fait tout aussi important, les participantes décrivaient la satisfaction au travail comme étant bénéficier d'une autonomie suffisante pour structurer les tâches et les activités au sein de leur journée de travail. L'aptitude des participantes à prendre des décisions et à déterminer leurs charges de travail de manière à répondre à leurs propres besoins et à ceux de leurs patients et proches révélait le degré de contrôle qu'elles exerçaient sur leur pratique. Une participante a ainsi déclaré :

Je peux choisir la manière dont je passe une bonne partie de ma journée, il y a les choses à faire mais de temps en temps je peux en mettre quelques-unes de côté et faire les choses que je veux faire pour ma propre satisfaction, la cerise sur le gâteau, en quelque sorte.

Insatisfaction à l'égard de la qualité de vie au travail. Les participantes faisaient état d'un éventail d'expériences auprès des

\begin{tabular}{|c|c|c|c|c|}
\hline \multicolumn{5}{|c|}{ Tableau 3. Questionnaire Obstacles (14 questions) } \\
\hline Question & $\begin{array}{l}\text { \% (n) Rép. } \\
\text { cochée }\end{array}$ & $\begin{array}{c}\text { Moyenne } \\
\text { (écart-type) }\end{array}$ & Médiane & Étendue \\
\hline $\begin{array}{l}\text { 1. Pas assez de } \\
\text { personnel infirmier }\end{array}$ & $100(9)$ & & & \\
\hline $\begin{array}{l}\text { 2. Patients trop } \\
\text { gravement malades }\end{array}$ & $33(3)$ & & & \\
\hline $\begin{array}{l}\text { 3. Conflit avec un } \\
\text { médecin }\end{array}$ & $33(3)$ & & & \\
\hline $\begin{array}{l}\text { 4. Le médecin } \\
\text { a découragé la } \\
\text { communication } \\
\text { avec les proches }\end{array}$ & $22(2)$ & & & \\
\hline $\begin{array}{l}\text { 5. Manque de } \\
\text { soutien de la part du } \\
\text { personnel infirmier/ } \\
\text { de travail social }\end{array}$ & $11(1)$ & & & \\
\hline $\begin{array}{l}\text { 6. À l'extérieur du } \\
\text { champ de pratique }\end{array}$ & $33(3)$ & & & \\
\hline $\begin{array}{l}\text { 7. La politique en } \\
\text { matière de visites } \\
\text { limite le temps } \\
\text { passé }\end{array}$ & $0(0)$ & & & \\
\hline $\begin{array}{l}\text { 8. Les proches ne } \\
\text { rendent pas visite/ } \\
\text { n'appellent pas }\end{array}$ & $22(2)$ & & & \\
\hline $\begin{array}{l}\text { 9. Manque de } \\
\text { communication avec } \\
\text { les professionnels }\end{array}$ & $56(6)$ & & & \\
\hline $\begin{array}{l}\text { 10. Proches en } \\
\text { colère }\end{array}$ & $67(6)$ & & & \\
\hline $\begin{array}{l}\text { 11. Attentes non } \\
\text { réalistes }\end{array}$ & $78(7)$ & & & \\
\hline $\begin{array}{l}\text { 12. Difficultés } \\
\text { personnelles avec } \\
\text { les proches }\end{array}$ & $44(4)$ & & & \\
\hline $\begin{array}{l}\text { 13. Manque de local } \\
\text { où communiquer } \\
\text { en privé }\end{array}$ & $78(7)$ & & & \\
\hline $\begin{array}{l}\text { 14. Problèmes de } \\
\text { langue }\end{array}$ & $78(7)$ & & & \\
\hline $\begin{array}{l}\text { Total de l'échelle } \\
\text { Obstacles }\end{array}$ & & $6,55(3,47)$ & 6,00 & 1 à 12 \\
\hline
\end{tabular}


patients et de leurs proches qui abaissaient leur qualité de vie au travail, notamment le fait de devoir s'occuper de patients/familles aux besoins complexes et reconnaître leur manque de compétence ou de formation face à certains enjeux liés aux patients/proches :

Les gens qui ont besoin de counseling, je les signale, oui, on a besoin ... de quelqu'un qui puisse travailler à mes côtés et se charger de ce qui dépasse mon champ de compétence et qui m'appuie. Et c'est ce que je crois, qu'il y a le soutien général que tu peux fournir à ton patient moyen mais je crois qu'il importe de réaliser nos limites et d'identifier ceux de nos patients qui ont besoin d'une aide dépassant ce que nous pouvons offrir et je pense que parfois nous nous engageons trop avant sans réaliser que ... mais il faut que nous nous reprenions et que nous les aiguillions vers quelqu'un d'autre ... nous désirons venir en aide aux gens, c'est la raison pour laquelle nous avons choisi les soins infirmiers, nous voulons satisfaire les gens, nous savons qu'ils traversent une rude épreuve, nous voulons la rendre aussi supportable que possible.

Bien que les participantes reconnaissent leurs limites, il leur arrivait parfois d'éprouver un sentiment d'impuissance et de frustration parce qu'il leur était impossible d'aborder efficacement les problèmes liés aux patients/familles, et jugeaient fréquemment que la qualité de leurs soins était mise en péril, comme quelques participantes l'ont si bien exprimé :

... Mais on ne peut s'empêcher de penser à des cas négatifs où le mari et la femme ne s'entendent pas entre eux et où il y avait toujours des conflits quand ils venaient à la clinique ... Ils venaient déballer leurs problèmes et ils se crêpaient le chignon dans la salle d'examen ... Cela arrive assurément et c'est une chose pour laquelle nous avons besoin d'aide et de soutien pour apprendre à aborder le problème.

La majorité des participantes mentionnaient de nombreux aspects de la détresse morale, notamment trouver un juste équilibre entre les besoins des patients/proches et leurs propres besoins en tant qu'infirmières; faire face aux désaccords d'opinion avec les patients, les proches ou les médecins relativement au plan de soins des patients; se demander si les patients ou leurs proches sont réellement tenus au courant du plan de traitement; et défendre les intérêts des patients vis-à-vis des décisions de la famille concernant le traitement ou les soins :

Parce que si tout ce que tu fais c'est d'être là et de les brancher à leur traitement et de leur demander comment ils se sentent et que tu t'en vas et passes immédiatement à un autre patient, tu n'as pas l'impression d'entendre réellement ce qu'ils te disent sur ce qu'ils éprouvent. Je crois donc que le temps est le facteur le plus important, pouvoir passer du temps avec eux.

... il y a de rares fois où nous nous demandons vraiment si le patient a été mis entièrement au courant et il y a donc conflit là ... sait-il vraiment qu'il peut y mettre un terme [au traitement], ou tu sais que leurs effets secondaires sont si horribles que tu te demandes si quelqu'un les a avertis qu'ils peuvent arrêter [le traitement] ou peut-être que la petite vieille qui est atteinte de démence, sait-elle vraiment ce qui se passe ici, ou bien est-ce qu'elle a été amenée ici par sa fille ou par son fils parce qu'ils ne veulent pas que maman les quitte.

Et parfois, il s'agit quelquefois d'intervenir en faveur du patient au sujet de sa famille .... Tu sais, leur dire savent-ils vraiment qu'ils ont besoin de faire ça?

Les infirmières n'hésitaient jamais à placer les besoins des patients et des proches au-dessus des leurs, en dépit du fait que ces divergences dans les besoins exacerbaient les contraintes du lieu de travail. Les autres cas de détresse morale cernés par les infirmières étaient les situations où les exigences, besoins ou attentes des patients/familles dépassaient les ressources des infirmières ou leur aptitude à répondre efficacement aux besoins des patients en soins de santé :

Certains individus font beaucoup de recherche sur Internet et ils arrivent pensant savoir ce qu'ils sont en droit d'attendre de nous et ils sont, je pense, un peu déçus par ce que nous leur offrons, je ne sais pas [...] ce qu'ils attendent vraiment de nous mais ce n'est certainement pas ce que nous leur donnons, davantage de temps est, je crois, ce qu'ils attendent de nous. Mais le temps nous manque et il n'y a pas d'intimité.

\section{Discussion}

Dans cette étude pilote, les limites se rapportent au recrutement et au faible échantillon de participantes dans deux milieux cliniques de cancérologie. Dans ce petit échantillon choisi à dessein, il est probable que les infirmières souhaitant participer aux groupes de discussion sur les relations infirmières-proches de patients aient introduit un biais dans leurs réponses, ce qui rend impossible toute généralisation des conclusions de l'étude aux infirmières œuvrant dans d'autres milieux cliniques ou centres de cancérologie. Cependant, les questions posées dans le cadre de l'entrevue ont été bien reçues par les participantes qui ont donné des réponses riches et variées au sujet de leurs expériences de travail auprès des familles de patients dans divers domaines cliniques. Après l'entrevue de groupe et l'invitation de l'infirmière chercheuse à donner leur rétroaction, les participantes ont déclaré avoir trouvé acceptables les questions des questionnaires et avoir pu y répondre sans trop de difficulté. Du fait de la faible taille de l'échantillon, le coefficient de fiabilité alpha de Cronbach n'a pas été calculé pour les échelles Activités, Obstacles et Satisfaction au travail. Une observation intéressante est que l'infirmière chercheuse devait fréquemment rediriger l'attention des participantes sur les soins aux proches plutôt que sur ceux aux patients. Cela laisse à penser que la satisfaction des besoins des patients était au centre des préoccupations des infirmières et que ceux-ci leur venaient plus facilement à l'esprit que de déterminer la manière dont elles abordaient les besoins des proches en particulier. McLeod, Tapp, Moules et Campbell (2010) ont également signalé que les infirmières de leur étude voyaient dans la famille le contexte de vie des patients qui les aidait à prodiguer des soins aux patients adaptés à la situation de ces derniers. Par contre, une fois qu'on leur avait demandé de se concentrer sur les soins offerts aux proches, les participantes ont fourni des réponses riches et approfondies aux questions de l'entrevue.

Malgré les écarts importants en termes d'années d'expérience dans la profession infirmière et d'années de travail au centre de cancérologie, toutes les infirmières décrivaient leurs diverses activités visant à répondre aux besoins des proches comme faisant partie des soins qu'elles dispensent. Les participantes parlaient de la valeur de l'écoute active, de l'empathie et du respect du principe selon lequel les membres de la famille et le patient forment une «unité de soins ». Elles étaient sensibilisées à la complexité des relations entre les patients et leurs proches, des défis que doivent surmonter les familles aux prises avec un diagnostic de cancer et des différences sur le plan des réponses au traitement et de l'adaptation chez les patients et leurs proches du fait de l'origine ethnique, de l'âge et de l'orientation sexuelle. Les participantes ont également expliqué que les familles avaient souvent leurs propres attentes en matière de soins en cancérologie. Leahey et Harper-Jacques (1996) ont nommé ce phénomène «interface entre le système patient-famille et le système infirmier» (p. 134, traduction libre), une constatation appuyée par les récentes conclusions d'ordre qualitatif avancées par McLeod, Tapp, Moules et Campbell (2010). Les participantes manifestaient une vive curiosité relativement à la vie des patients et de leurs proches, ce qui dénote l'importance qu'elles accordent à la facette humaine des soins oncologiques. Elles parlaient de normalisation du vécu de la maladie, un phénomène également décrit par McLeod, Tapp, Moules 
et Campbell (2010), et désigné par Morse (1991), dans les écrits déjà anciens, comme une relation thérapeutique où l'infirmière considère la personne malade à la fois comme un patient et comme une «personne ayant une vie 'à l'extérieur'» (p. 458, traduction libre). Les participantes promouvaient des relations non hiérarchiques, comme il ressortait des descriptions qu'elles faisaient de la manière dont elles partageaient leur être et valorisaient ou reconnaissaient les contributions des patients atteints de cancer et de leurs proches aux soins qu'elles prodiguaient. Madsen (2009) rangerait cette approche sous le vocable «souligner la sagesse des clients» [traduction libre], une approche où les infirmières ne privilégient pas leurs connaissances professionnelles par rapport à celles des patients et des proches. Dans une même optique, Leahy et Harper-Jones (1996) diraient que ces infirmières se sont munies d'une «lentille non saturée par les problèmes» [traduction libre], pour parler des choses que les familles et les patients font bien alors qu'ils sont confrontés au cancer. Les activités de dispensation de soins effectuées par les infirmières de l'étude sont des comportements de communication empreinte d'empathie (Kruijver, Kerkstra, Bensing \& van de Wiel, 2000). Se donnant pour but de réconforter les patients et leurs proches et d'appuyer les atouts familiaux, les participantes à l'étude ajoutaient une note particulière et prenaient le temps de parler de la vie en général et des événements agréables jalonnant la vie personnelle des patients et de leurs proches.

Les participantes s'efforçaient de maximiser les soutiens des familles en matière d'information et de vie affective en les encourageant à adopter une démarche de questionnement, en leur fournissant des renseignements sur la manière de s'y retrouver dans le système des soins contre le cancer, en étant sensibles à leur phase d'adaptation, en les rassurant sur l'équipe de soins de santé et enfin, en éprouvant leur douleur. Les infirmières étaient à l'aise lorsqu'il s'agissait d'enseigner aux patients et aux proches des techniques de communication de base afin qu'ils puissent jouer un rôle actif et de promouvoir chez eux l'autonomie sociale et l'assertivité lorsqu'ils posent leurs questions au cours de la consultation médicale et se mettent en quête de ressources dans le système de soins global. Les infirmières n'ignoraient pas la nature oppressive et limitative du système de soins contre le cancer, et elles intervenaient aux côtés des familles afin d'attirer l'attention sur les besoins des patients et de leurs soignants naturels. De manière générale, ces infirmières décrivaient la gamme complète de leurs interactions et interventions, laquelle traduisait leur engagement à l'égard de leurs relations avec les patients et leurs proches tout en s'évertuant à atteindre un juste équilibre entre les priorités dégagées par leur milieu de travail, pour le bien des patients et des proches, et en établissant les limites de leur espace professionnel, pour leur propre bien. Quoique ces participantes travaillent dans un système axé sur la maladie, elles n'en définissaient pas moins le vécu des patients et de leurs proches dans le contexte de leur existence quotidienne. Malgré l'absence d'une démarche officielle dans leur établissement en vue d'évaluer les besoins, circonstances, forces et objectifs des familles, les participantes décrivaient leur implication active auprès des proches afin de dégager leur capacité d'adaptation et d'accès aux services pour répondre à leurs propres besoins et à ceux des patients.

Il est intéressant de noter que la satisfaction des besoins spirituels ou religieux des familles était la question que le plus faible nombre des infirmières participant à l'étude-67\%-ont mentionnée avoir fait. Cependant, il est possible que ce résultat soit conforme aux travaux de Taylor (2006) selon qui, chez les patients et leur famille, le besoin d'assistance spirituelle au niveau des soins en cancérologie était seulement modéré et variait grandement. De plus, Taylor (2006) avançait que les besoins spirituels des patients et de leurs proches étaient représentés par diverses manières d'être dont adopter une attitude positive, aimer autrui, rechercher la signification et enfin, le rapport avec Dieu. En conclusion, Taylor conseillait aux infirmières de faire preuve de prudence lorsqu'elles communiquaient avec les patients en vue de dispenser des soins spirituels et ce, uniquement dans le cas de patients et de proches ayant exprimé ouvertement un tel besoin, c'est-à-dire ceux qui font face à une hospitalisation ou à un cancer menaçant leur existence.

Du point de vue quantitatif, les obstacles perçus comme les plus courants étaient : pas assez de personnel infirmier, attentes non réalistes des familles, manque d'espaces privés, problèmes de langue et enfin, faire face aux proches en colère. Dans un même ordre d'idées, Bakker et ses collègues (2010) ont récemment signalé que 39 pour cent de leur échantillon d'infirmières rapportaient que le personnel ne comprenait pas suffisamment d'infirmières pour fournir des soins de qualité. Dans les contextes de traitement ou de chimiothérapie où un accent particulier est mis sur les tâches, les compétences et la nature technique des soins aux personnes atteintes de cancer, les réponses qualitatives des participantes révélaient les défis qu'elles devaient surmonter afin d'assurer un juste équilibre entre la prestation de ces types d'interventions infirmières et celle des soins émotionnels aux patients et à leurs proches. Tout comme dans l'étude de Mcilfatrick et collaborateurs (2006), les participantes exprimaient le vif besoin qu'elles ressentaient «d'être aux côtés » des patients et de leurs proches, notamment dans le cas des patients recevant leur tout premier traitement. Afin de pouvoir répondre aux besoins des patients et de leurs proches en dépit des contraintes de temps, les infirmières prenaient l'initiative et cherchaient des solutions en comptant sur le soutien d'autres infirmières tandis qu'elles étaient présentes aux côtés de patients et de leurs proches tous angoissés à l'idée de passer par le «premier cycle». Kelly (1998) a inventé le terme «soins du monde réel» [traduction libre] pour décrire cette démarche où les infirmières alliaient l'établissement de relations, d'une part, à l'administration des traitements aux patients, d'autre part. Le manque d'espace avait également été relevé par Mcilfatrick et ses collaborateurs (2006) lesquels constataient que les infirmières n'avaient pas nécessairement la possibilité de développer des relations avec les patients et leurs proches lorsque ceux-ci hésitent à exprimer leurs préoccupations à haute voix dans des zones de traitement par trop ouvertes.

Un autre obstacle majeur était de devoir faire face à la colère des proches, tel qu'il a été rapporté dans d'autres études (Beckstrand \& Kirschoff, 2005; Beckstrand, Smith, Heaston \& Bond, 2008; Heaston, Beckstrand, Bond \& Palmer, 2006). Toutefois, plusieurs infirmières chevronnées ont rapporté la persévérance dont elles font preuve en vue d'aider à résoudre ou à clarifier les idées fausses et à travailler sur les émotions jusqu'à l'obtention des réponses ou comportements désirés. D'autres participantes ont souligné l'importance du travail en équipe grâce auquel elles pouvaient compter les unes sur les autres quand elles étaient confrontées à des contraintes de temps ou à des défis liés à des enjeux personnels au sein des familles. Des sentiments de frustration et la nécessité de recevoir du soutien survenaient lorsque les participantes remettaient le plan de traitement en question et si les proches et les patients avaient une compréhension suffisamment bonne de la pertinence du plan de traitement quant à leurs propres buts et valeurs et quant à leur aptitude à composer avec les exigences du plan.

La frustration signale la détresse morale que les infirmières éprouvent lorsque leurs valeurs morales sont mises en péril par les restrictions existant dans leur environnement de travail, par les obstacles liés à leur établissement et par les conflits interpersonnels (Corley et al., 2001; Peter, Macfarlene \& O'Brien-Pallas, 2004; Shepard, 2010; Zuzelo, 2007). Certaines interventions cliniques laissaient entrevoir la présence possible de détresse morale chez les infirmières. Par exemple, quand les infirmières se demandaient si les patients et leurs proches avaient été vraiment informés au sujet des soins, elles se sentaient obligées de défendre les intérêts des patients en demandant que les médecins évaluent de nouveau les besoins, valeurs et buts des patients. Cette conclusion est corroborée par l'étude qualitative de Fitch (1998) où les infirmières décrivaient le 
rôle de médiation qu'elles jouaient dans les conflits entre membres de la famille et patients relativement aux buts des soins. Quelques infirmières ont cerné le besoin qu'elles avaient de disposer de davantage de temps durant leurs journées de travail pour assister à des séances d'éducation et de soutien où elles se sentent en sûreté pour divulguer et valider leur démarche de résolution de problèmes dans le cadre de situations à fortes implications morales ou d'une dynamique complexe entre patients et proches. Les infirmières ayant participé à l'étude de Bakker et collègues (2010) ont mentionné un besoin similaire en matière de possibilités de perfectionnement professionnel et d'éducation permanente dans leur milieu de travail.

Les plus hauts taux de satisfaction à l'égard de la qualité de la vie professionnelle et des relations avec les familles concernaient les comportements sur lesquels les infirmières exerçaient un contrôle et qu'elles percevaient comme étant utiles, tels que la satisfaction des besoins physiques des proches. Les infirmières décrivaient les initiatives auxquelles elles participaient en vue de trouver dans leur contexte clinique des endroits d'un confort physique adéquat et suffisamment privé où les patients et leurs proches pouvaient discuter en toute intimité de leurs inquiétudes et questions. Pour l'infirmière, l'autonomie dont elle jouit au sein de son rôle pour répondre aux besoins des proches est d'une grande valeur au regard de la satisfaction au travail, un concept qui trouve écho dans les écrits scientifiques (Bakker, Fitch, Green, Butler \& Olson, 2006; Cummings et al., 2008; Zangaro \& Soeken, 2007). Il est manifeste que les participantes réagissaient positivement à un environnement de travail autonome, certes, mais aussi collégial qui les appuyait dans leurs soins aux patients et aux proches. Le niveau de satisfaction était à son plus faible lorsqu'il s'agissait de répondre aux besoins émotionnels des proches. Une dynamique familiale difficile forçait parfois les infirmières à prendre leurs distances vis-à-vis des patients ou des proches en colère. Dans la présente étude, les infirmières qui exprimaient le moins de confiance au niveau de l'exécution de diverses tâches de communication avaient tendance à rester à l'écart des situations familiales désagréables ou chargées d'émotion. Des infirmières disaient se sentir coupables et passer des nuits blanches lorsque les besoins des proches et des patients n'étaient pas satisfaits de manière adéquate. Toutefois, ces infirmières dirigeaient comme il se doit ces familles vers l'attention compétente et appropriée d'autres membres de l'équipe de soins tels qu'une travailleuse sociale ou un aidant spirituel. Il arrivait, des fois, que les opinions personnelles des infirmières soient diamétralement opposées à celles des patients, proches or médecins et que, d'autres fois, les participantes s'inquiètent de ne pas pouvoir répondre aux attentes des patients et de leurs familles. Dans un même ordre d'idées, Sivesind, Parker, Cohen, DeMoor, Bumbaugh, Throckmorton et Baile (2003) ainsi que Rask, Jensen, Andersen et Zachariae (2009) ont découvert que la résolution de plaintes physiques et la satisfaction des besoins physiques étaient ce qui posait le moins problème mais que les infirmières trouvent désirable de pouvoir répondre aux besoins émotionnels en manifestant des compétences de communication optimales les aidant à composer avec les conflits entourant les décisions relatives au traitement, à gérer les proches surprotecteurs et à intervenir auprès des proches animés de colère.

\section{Conclusions et implications}

Cette étude multi-méthodes avait pour but d'explorer les relations infirmière-proches de patients dans un milieu de soins ambulatoires en oncologie et de déterminer le rapport que ces relations entretiennent avec la satisfaction des infirmières envers leur qualité de vie au travail. Les participantes décrivaient volontiers l'incidence de leur environnement de travail sur leur état de préparation et leur volonté de s'occuper de l'impact du cancer sur les membres de la famille. Les participantes ne se limitaient pas à des modèles de soins axés sur les tâches mais s'impliquaient aussi dans des soins encourageant la participation en vue de répondre aux besoins des proches. Cependant, les infirmières de l'étude signalaient que ce qui leur posait le plus problème était la satisfaction des besoins émotionnels dans le cadre de situations familiales difficiles. Les infirmières ont besoin de soutien pour surmonter les obstacles à l'adoption d'une démarche relationnelle avec les proches en leur permettant de se concentrer sur leur aptitude à établir et développer des relations afin de rehausser les «unités de soins» patients-proches ainsi que leur satisfaction envers leur qualité de vie au travail. Les participantes ont offert de nombreuses recommandations pratiques aux administrateurs en vue d'améliorer leur degré de satisfaction dans leurs relations avec les proches dans leur milieu de soins ambulatoires.

La première recommandation portait sur des programmes de soutien où les infirmières pourraient discuter des cas difficiles et se soutenir les unes les autres afin de réduire les incertitudes liées aux soins dispensés aux proches et de réduire la détresse morale. En deuxième lieu, les infirmières recommandaient d'accroître le perfectionnement du personnel afin de rehausser ses connaissances et compétences en soins axés sur la famille, notamment pour ce qui est d'aborder les attentes des proches, les conflits patients-proches, la colère familiale et les besoins émotionnels. En troisième lieu, il est recommandé que les gestionnaires aident les infirmières à trouver un meilleur équilibre entre les aspects techniques des soins infirmiers (p. ex. le fonctionnement efficace de la clinique et l'administration de la chimiothérapie) et les aspects relationnels des soins aux patients et aux proches dans le milieu de travail. Par exemple, les infirmières ont parlé à maintes reprises de la nécessité d'avoir des locaux privés et d'augmenter l'effectif afin que les infirmières aient davantage le temps de faire de l'écoute active et de manifester leur empathie et leur respect envers les patients et les soignants membres de la famille. En quatrième lieu, malgré les contraintes de temps, d'espace et de ressources liées à leur environnement de travail, ces infirmières exprimaient la fierté qu'elles ressentaient envers leur aptitude à répondre aux besoins des patients et de leurs familles. Les environnements qui habilitent les infirmières en favorisant chez elles une autonomie maximale dans leur pratique, les encouragent ainsi à exercer pleinement leur pratique (Bakker et al., 2006). Étant données les lacunes dégagées, en théorie, sur la qualité de la vie professionnelle des infirmières concernant leurs relations avec les proches, la première mesure logique serait que des chercheuses mènent des entrevues auprès d'infirmières administratrices sur l'environnement de travail et les philosophies du lieu de travail liées aux soins axés sur la famille qui soutiennent ou au contraire entravent la capacité des infirmières à donner des soins aux proches des patients atteints de cancer et à développer des relations avec eux.

\section{Remerciement}

Ces travaux de recherche ont été soutenus par une subvention de recherche de l'ACIO/CANO et une subvention de Chercheur boursier de l'Institut nnational du cancer du Canada, les fonds de la Société canadienne du cancer ayant été versés à la première auteure. Nous souhaitons également remercier la $D^{\text {re }}$ Carmen Loiselle pour la précieuse assistance qu'elle a apportée à la planification de ce projet et pour ses commentaires constructifs sur la version préliminaire de

\section{Références}

Agence de la santé publique du Canada. (2004). Rapport d'étape sur

la lutte contre le cancer au Canada. Ottawa: Santé Canada.

Association canadienne des infirmières en oncologie. (2001). Normes de soins, rôles infirmiers en oncologie et compétences relatives aux rôles infirmiers. Kanata, ON: Association canadienne des infirmières en oncologie. 
Bakker, D., Conlon, M., Fitch, M., Green, E., Butler, L., Olson, K., \& Cummings, G. (2010). Canadian oncology nurse work environments: Part I. Nursing Leadership, 22(4), 50-68.

Bakker, D., Fitch, M.I., Green, E., Butler, L., \& Olson, K. (2006). Les soins infirmiers en oncologie : la recherche d'un équilibre dans un système de soins en évolution. Revue canadienne de soins infirmiers en oncologie, 16(2), 88-98.

Beckstrand, R.L., \& Kirchoff, K.T. (2005). Providing end of life care to patients: Critical care nurses' perceived obstacles and supportive behaviors. American Journal of Critical Care, 14(5), 395-403.

Beckstrand, R.L., Smith, M.D., Heaston, S., \& Bond, A.E. (2008). Emergency nurses' perceptions of size, frequency, and magnitude of obstacles and supportive behaviors in end-of-life care. Journal of Emergency Nursing, 34(3), 290-300.

Corley, M., Elswick, R., Gorman, M., \& Clor, T. (2001). Development and evaluation of a moral distress scale. Journal of Advanced Nursing, 33, 250-256.

Cummings, G.C., Olson, K., Hayduk, L., Bakker, D., Fitch, M., Green, E., ... Conlon, M. (2008). The relationship between nursing leadership and nurses' job satisfaction in Canadian oncology work environments. Journal of Nursing Management, 16, 508518.

Downey, L., Engelberg, R.A., Shannon, S.E., \& Curtis, J.R. (2006). Measuring intensive care nurses' perspectives on family-centered end-of-life care: Evaluation of 3 questionnaires. American Journal of Critical Care, 15(6), 568-579.

Eriksson, E. (2001). Caring for cancer patients: relative's assessments of received care. European Journal of Cancer Care, 10, 48-55.

Fitch, M.I. (1998). La qualité de vie en oncologie: les perceptions, valeurs et comportements des infirmières. Revue canadienne de soins infirmiers en oncologie, 8(1), 31-39.

Flanagan, J. (2001). Clinically effective cancer care: Working with families. European Journal of Oncology Nursing, 5(3), 174-179.

Greco, P., Laschinger, H.K., \& Wong, C. (2006). Leader empowering behaviours, staff nurse empowerment and work engagement/ burnout. Nursing Leadership, 19 (4), 41-56.

Heaston, S., Beckstrand, R.L., Bond, A.E., \& Palmer, S.P. (2006). Emergency nurses' perceptions of obstacles and supportive behaviors in end-of-life care. Journal of Emergency Nursing, 32(6), 477-485.

Hobbs, S.F., \& Sodomka, P.F. (2000). Developing partnerships among patients, families, and Staff at the Medical College of Georgia Hospital and Clinics. Joint Commission on Accreditation of Healthcare Organizations, 26(5), 268-276.

Isikhan, V., Comez, T., \& Danis, M.Z. (2004). Job stress and coping strategies in health care professionals working with cancer patients. European Oncology Nursing Society, 8, 234-244.

Kelly, D. (1998). Caring and cancer nursing: Framing the reality of using selected social science theory. Journal of Advanced Nursing, 28, 728-736.

Kruijver, I.P., Kerkstra, A., Bensing, J.M., \& van de Wiel, H.B. (2000). Nurse-patient communication in cancer care: A review of the literature. Cancer Nursing, 23(1), 20-31.

Laschinger, H.K., \& Finegan, J. (2005). Empowering nurses for work engagement and health in hospital settings. Journal of Nursing Administration, 35(10), 439-49.

Laschinger, H.K., Almost, J., \& Tuer-Hodes, D. (2003). Workplace empowerment and magnet hospital characteristics: Making the link. Journal of Nursing Administration, 33(7-8), 410-22.

Leahy, M., \& Harper-Jacques, S. (1996). Family-nurse relationships: Core assumptions and clinical implications. Journal of Family Nursing, 2(2), 133-151.

Lincoln, Y.S., \& Guba, E.G. (1985). Naturalistic inquiry. Beverly Hills, CA: Sage Publications, Inc.
Lindholm, L., Makela, C., Rantanen-Siljamaki, S., \& Nieminen, A.L. (2007). The role of significant others in the care of women with breast cancer. International Journal of Nursing Practice, 13, 173-181.

Madsen, W.C. (2009). Collaborative helping: A practice framework for family-centered services. Family Process, 48(1), 103-116.

McGillis Hall, L., Doran, D., \& Pink, L. (2008). Outcomes of interventions to improve hospital nursing work environments. The Journal of Nursing Administration, 38 (1), 40-46.

Mcilfatrick, S., Sullivan, K., \& McKenna, H. (2006). What about the carers?: Exploring the experience of caregivers in a chemotherapy day hospital setting. European Journal of Oncology Nursing, 10, 294-303.

McLeod, D.L., Tapp, D.M., Moules, N.J., \& Campbell, M.E. (2010). Knowing the family: Interpretations of family nursing in oncology and palliative care. European Journal of Oncology Nursing, 14, 93-100.

Morse, J.M. (1991). Negotiating commitment and involvement in the nurse-patient relationship. Journal of Advanced Nursing, 16, 455-468.

O’Brien-Pallas, L., Tomblin-Murphy, G., White, S., Hayes, L., Baumann, A., Higgin, A. ... Wang, S. (2005). Building the future: An integrated strategy for nursing human resources in Canada. Research synthesis report. Ottawa: The Nursing Sector Study Corporation. Retrieved from http://www.buildingthefuture.ca/ e/study/phase1/reports/Research-Synthesis-Report.pdf

Patton, M. (2002). Qualitative research \& evaluation methods $\left(3^{\text {rd }}\right.$ ed.). Thousand Oaks, CA: Sage.

Peter, E.H., Macfarlene, A.V., \& O’Brien-Pallas, L.L. (2004). Analysis of the moral habitability of the nursing work environment. Journal of Advanced Nursing, 47(4), 356-367.

Priest, A. (2006). What's ailing our nurses? A discussion of the major issues affecting nursing human resources in Canada. Retrieved from http://www.chsrf.ca/research_themes/pdf/what_sailing ourNurses-e.pdf

Rask, M.T., Jensen, M.L., Andersen, J., \& Zachariae, R. (2009). Effects of an intervention aimed at improving nurse-patient communication in an oncology outpatient clinic. Cancer Nursing, 32(1), E1-11.

Shepard, A. (2010). Moral distress: A consequence of caring. Clinical Journal of Oncology Nursing, 14(1), 25-27.

Sivesind, D., Parker, P.A., Cohen, L., DeMoor, C., Bumbaugh, M., Throckmorton, T., ... Baile, W. (2003). Communicating with patients in cancer care: What areas do nurses find most challenging? Journal of Cancer Education, 18(4), 202-209.

Société du cancer du Canada-Comité directeur. (2010). Statistiques canadiennes sur le cancer 2010. Toronto: Société du cancer du Canada.

Strauss, A., \& Corbin, J. (1998) Basics of Qualitative Research: Techniques and Procedures for Developing Grounded Theory. London: Sage.

Tamayo, G.J., Broxson, A., Munsell, M., \& Cohen, M.Z. (2010). Caring for the caregiver. Oncology Nursing Forum, 37(1), E50-56.

Taylor, E.J. (2006). Prevalence and associated factors with spiritual needs among patients with Cancer and their family caregivers. Oncology Nursing Forum, 33(4), 725-735.

Torgerson, R. (2007). Not there yet: Improving the working conditions of Canadian nurses. Ottawa: Office of Nursing Policy, Health Canada.

Vivar, C.G., Canga, N., Canga, A.D., \& Arantzamendi, M. (2009). The psychosocial impact of recurrence on cancer survivors and family members: A narrative review. Journal of Advanced Nursing, 65(4), 724-736.

Zangaro, G.A., \& Soeken, K.L. (2007). A meta-analysis of studies of nurses' job satisfaction. Research in Nursing \& Health, 30, 445-458.

Zuzelo, P.R. (2007). Exploring the moral distress of registered nurses. Nursing Ethics, 14(3), 344-359. 